This document is the Accepted Manuscript version of a Published Work that appeared in final form in Chemistry of Materials:

Saeed-Uz-Zaman Khan, Giacomo Londi, Xiao Liu, Michael A. Fusella, Gabriele D’Avino, Luca Muccioli, Alyssa N. Brigeman, Bjoern Niesen, Terry Chien-Jen Yang, Yoann Olivier, Jordan T. Dull, Noel C. Giebink, David Beljonne, Barry P. Ran.

Multiple charge transfer states in Donor-acceptor heterojunctions with large frontier orbital energy offsets.

Chemistry of Materials, Volume 31, Issue 17, 10 September 2019, Pages 6808-6817.

(C) 2019 American Chemical Society after peer review and technical editing by the publisher.

To access the final edited and published work see

http://dx.doi.org/10.1021/acs.chemmater.9b01279 


\title{
Multiple Charge Transfer States in Donor-Acceptor Heterojunctions with Large Frontier Energy Offsets
}

Saeed-Uz-Zaman Khan ${ }^{1}$, Giacomo Londi ${ }^{2}$, Xiao Liu ${ }^{1}$, Michael Fusella ${ }^{1}$, Gabriele D'Avino ${ }^{3}$, Luca Muccioli ${ }^{4,5}$, Alyssa N. Brigeman ${ }^{6}$, Bjoern Niesen ${ }^{7}$, Terry Chien-Jen Yang ${ }^{7}$, Yoann Olivier ${ }^{2}$, Noel C. Giebink ${ }^{5}$, David Beljonne ${ }^{2}$, and Barry P. Rand ${ }^{1,8}$

\footnotetext{
${ }^{1}$ Department of Electrical Engineering, Princeton University, Princeton, NJ, 08544, United States ${ }^{2}$ Laboratory for Chemistry of Novel Materials, University of Mons, Place du Parc 20, 7000 Mons, Belgium

${ }^{3}$ Institut Néel, CNRS and Grenoble Alpes University, F-38042 Grenoble, France

${ }^{4}$ Dipartimento di Chimica Industriale “Toso Montanari”, Università di Bologna, I-40136, Bologna, Italy ${ }^{5}$ Institut des Sciences Moléculaires, UMR 5255, University of Bordeaux, F- 33405 Talence, France ${ }^{6}$ Department of Electrical Engineering, The Pennsylvania State University, University Park, Pennsylvania 16802, USA

${ }^{7}$ Photovoltaics and Thin-Film Electronics Laboratory, Institute of Microengineering (IMT), Ecole Polytechnique Fédérale de Lausanne (EPFL), Maladière 71, Neuchâtel 2000, Switzerland

${ }^{8}$ Andlinger Center for Energy and the Environment, Princeton University, Princeton, New Jersey 08544, USA
}

\begin{abstract}
In this work we demonstrate several organic amorphous donor-acceptor systems that exhibit subbandgap features over a more than $2 \mathrm{eV}$ spectral range. An in-depth study of one of these systems, NPB-HAT-CN (NPB is $N, N^{\prime}$-di(1-naphthyl)- $N, N^{\prime}$-diphenyl-(1,1'-biphenyl)-4,4'-diamine and HAT-CN is 1,4,5,8,9,11-hexaazatriphenylenehexacarbonitrile), reveals that the broad subbandgap features are attributed to multiple electronic charge transfer (CT) state transitions, broadened by energetic disorder sourcing from the fluctuations of intra-molecular conformations and by the disordered intermolecular environment. These unique CT features originate from an unconventional donor and acceptor selection that reveals new insight about photocurrent generation and non-radiative recombination. Unlike materials employed in high performing organic solar cells, the materials studied here feature large optical energy gaps with very large frontier energy level offsets, creating high bandgap devices with low open-circuit voltage. In addition to multiple electronic CT levels, we reveal that the internal quantum efficiency of these multiple CT transitions are not constant, but photon energy dependent, and with emission that originates primarily from the second lowest electronic CT state implying slow (relative to radiative and nonradiative rates) internal conversion within the $\mathrm{CT}$ manifold. Overall, this class of donor-acceptor pairs provides an opportunity to probe CT states in unique ways to potentially unravel their role in carrier generation-recombination and energy loss mechanisms.
\end{abstract}




\section{Introduction}

With more than $15 \%$ power conversion efficiency recently achieved, ${ }^{1}$ and coupled with the ability to be grown on flexible substrates at a large scale with low-cost fabrication techniques, organic solar cells are making a strong case for energy conversion applications. Most advances in power conversion efficiency are the result of impressive material and interface engineering and innovation. However, due to the lack of a complete understanding and consensus of carrier generation and recombination processes as well as loss mechanisms at charge generating donor-acceptor (D-A) interfaces, ${ }^{2}$ the actual limits of organic solar cell performance remain unknown. In this context, intermolecular charge transfer (CT) excitations at D-A interfaces play an important role in the photophysical processes and energy losses of organic solar cells. ${ }^{3-6}$ In order to understand how carrier generation and recombination are affected by CT states, a better understanding of their origin and energetic distribution is crucial. More specifically, some key issues that are currently under inquiry include whether the carrier dissociation efficiency depends on incident photon energy ${ }^{3,7-9}$ or not, ${ }^{10,11}$ the origin of the broadening of the CT state line-shape (static and dynamic disorder), ${ }^{6,12-15}$ the possibility and origin of multiple CT states (electronic, vibronic, or morphology induced), ${ }^{16-20}$ and reciprocity of CT state absorption and emission spectra (thermal and non-thermal state distribution). ${ }^{21-24}$ One step towards addressing these issues is to identify organic D-A systems that display unique CT state characteristics, in order to probe CT state energies in novel ways.

The optical signature of CT states is often buried in the background signal of much more strongly absorbing Frenkel (i.e. intramolecular or localized) excitons, despite that multiple CT states are predicted to exist. ${ }^{25}$ While techniques that exploit anisotropy can reveal the CT state region underneath this background, ${ }^{26}$ such methods are effective only in the presence of crystalline order that results in sufficient dipole alignment at the D-A interface. In this work, we present several wide bandgap D-A systems consisting of both small molecule and polymer organic semiconductors with large frontier energy offsets (i.e. large energetic driving force for charge separation), resulting in ultra-broad CT state spectra. Despite varying molecular structure and energetics, these large offset heterojunctions display complex CT features that span more than $2 \mathrm{eV}$, well outside the Frenkel exciton region.

We then investigate the nature of these sub-bandgap features in detail through a comprehensive experimental and computational study of the D-A system of NPB:HAT-CN (NPB is $N, N^{\prime}$-di(1-naphthyl)- $N, N^{\prime}$-diphenyl-(1,1'-biphenyl)-4,4'-diamine and HAT-CN is 1,4,5,8,9,11-hexaazatriphenylenehexacarbonitrile). Ultra-sensitive sub-bandgap absorption spectra reveal the existence of low energy CT states that do not contribute to photocurrent as probed via spectrally-resolved external quantum efficiency (EQE) measurements, despite having comparable (relative to higher energy CT states) absorption strength. Through spectral absorption, EQE, photoluminescence (PL) measurements, and combined molecular dynamics (MD) simulations and electronic structure calculations, we confirm the existence of multiple electronic CT states at these heterojunctions, which are broadened by nanoscale conformational and electrostatic variations. Finally, EQE measurements under applied voltage bias indicate that internal quantum efficiency (IQE) is strongly dependent on incident photon energy in these large 
offset heterojunction solar cells, providing an example of non-constant IQE. We attribute the reduced IQE of low energy CT states to their strong coupling to the ground-state, and hence strong non-radiative recombination.

\section{Results and Discussion}

In this work, we demonstrate five D-A systems that exhibit approximately $2 \mathrm{eV}$ wide sub-gap spectra, by blending wide optical energy gap materials with large offset energies. As donors, we used the small molecules NPB, 4,4',4"-tris[(3methylphenyl)phenylamino]triphenylamine (m-TDATA) and 4,4'-cyclohexylidenebis[ $N, N$ bis(4-methylphenyl)benzenamine] (TAPC) and the polymer poly(9-vinylcarbazole) (PVK). As acceptors, we employed either HAT-CN or 1,4,5,8-naphthalenetetracarboxylic dianhydride (NTCDA). All donor molecules here have optical gaps greater than $3 \mathrm{eV}$, while the acceptor molecules have approximately $4 \mathrm{eV}$ optical gap and large electron affinity (EA) (see Table S1). The energy gap between the highest occupied molecular orbital (HOMO) of the donor and lowest unoccupied molecular orbital (LUMO) of the acceptor is therefore significantly smaller than in typical high-performing organic solar cells. This leads to low open-circuit voltage $\left(V_{\mathrm{OC}}\right)$ and, notably, broad sub-bandgap spectral features as shown in Fig. 1 for co-evaporated bulk heterojunctions (BHJs), except for the sequentially deposited PVK/HAT-CN planar heterojunction (PHJ). Interestingly, the EQE features of all devices end at approximately $1.1 \mathrm{eV}$, despite considerable variations in their CT energy gap, IP(donor)-EA(acceptor), as inferred from measurements on the individual materials.

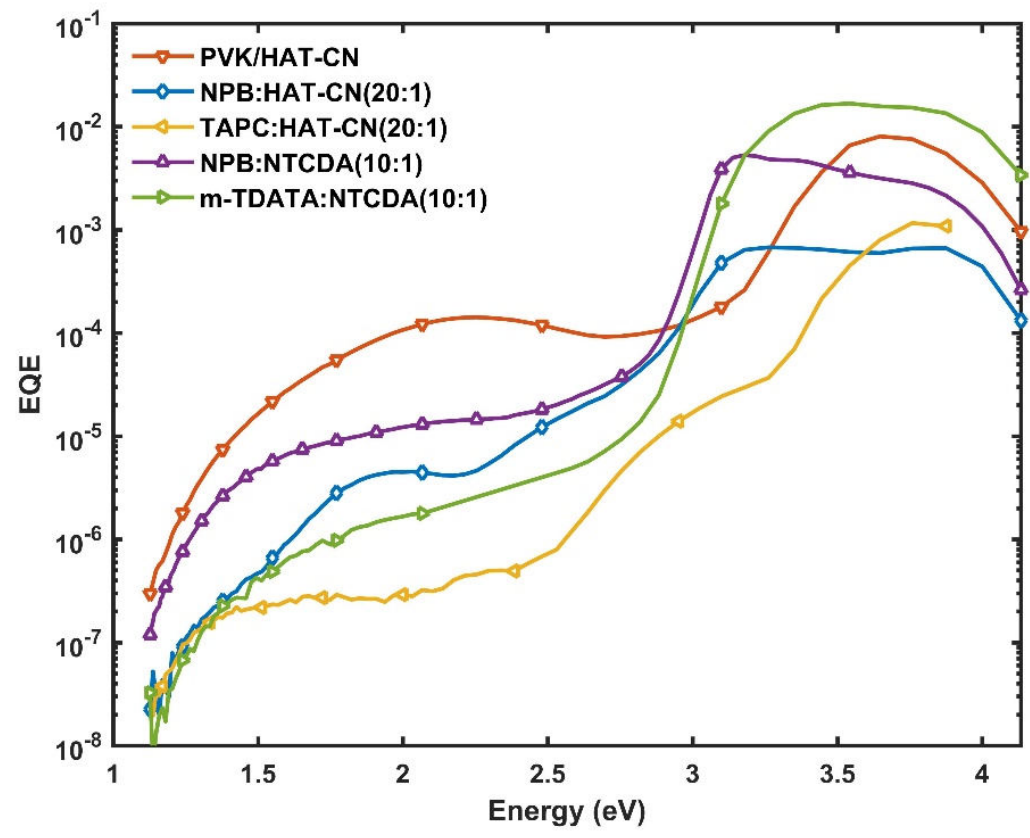

Figure 1. External quantum efficiency (EQE) spectra of organic bulk and planar heterojunction solar cells, exhibiting broad CT spectra extending to approximately $1.1 \mathrm{eV}$. 
To understand the nature of the broad sub-bandgap EQE features, we have conducted an in-depth investigation on the NPB-HAT-CN D-A system. The EQE spectra for NPB:HAT-CN BHJs with various D:A ratios are shown in Fig. 2. For the 20:1 D:A blend, there are three distinct sub-bandgap EQE features centered around 1.4, 2.0 and $2.8 \mathrm{eV}$ that match the absorption peaks in the photothermal deflection spectroscopy (PDS) spectrum, except that the lowest energy EQE shoulder is cut-off at around $1.1 \mathrm{eV}$. All three features in the EQE spectrum can then be attributed to photocurrent generation from direct CT absorption.

The comparison to a control device with NPB p-doped by the very strong oxidant $\mathrm{MoO}_{3}$ (see Fig. S1) provides additional information about the sub-gap features of NPB:HAT-CN BHJs. The PDS spectra of $\mathrm{MoO}_{3}$-doped NPB show two distinct polaron absorption peaks located at 0.9 and $2.6 \mathrm{eV}$, which do not exactly match the spectral features in Fig. 2. The low-energy tail of the NPB:HAT-CN PDS spectrum in Fig. 2 (dashed red line) seems to suggest the presence of the NPB cation in this blend, i.e. a slight doping. Moreover, we cannot exclude that the feature around $2.5-2.8 \mathrm{eV}$ in the EQE and PDS of NPB:HAT-CN presents a contribution from the corresponding polaron peak observed in the PDS spectrum of $\mathrm{MoO}_{3}$-doped NPB. An additional hint of the special role played by HAT-CN is that the EQE of $\mathrm{MoO}_{3}$-doped NPB single layer devices (Fig. S1) show no sub-gap signal.

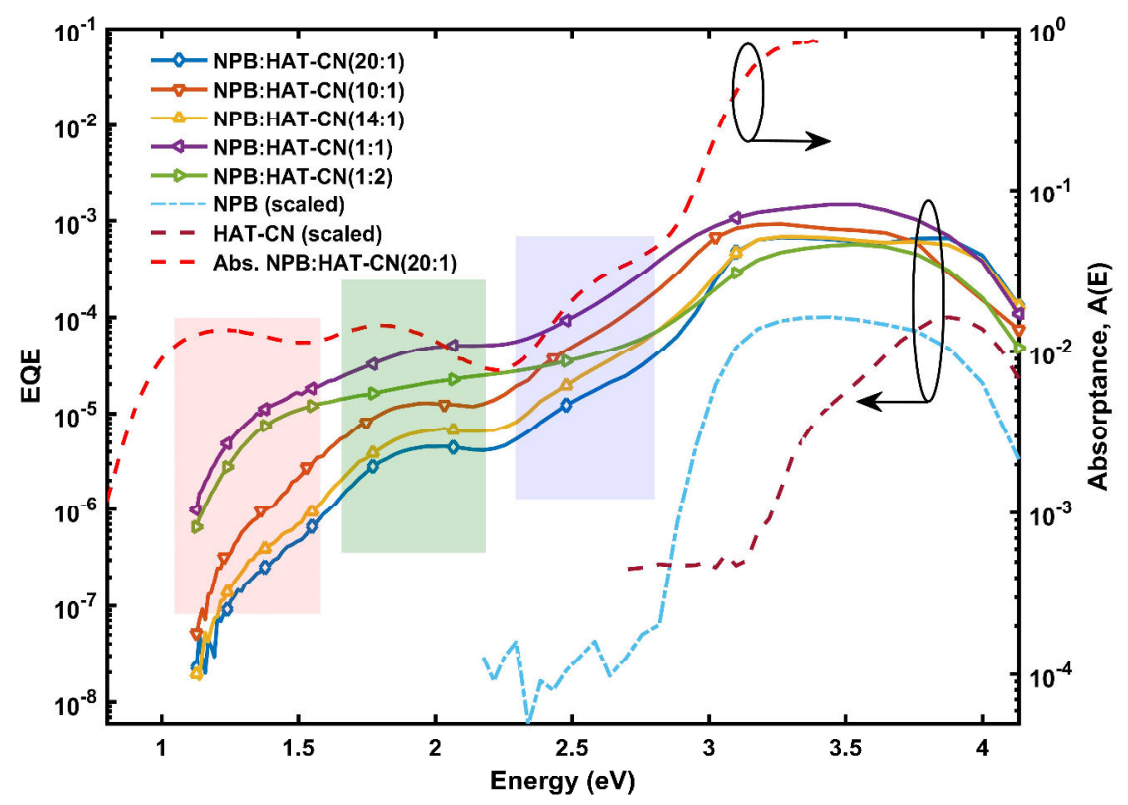

Figure 2. External quantum efficiency (EQE) spectra of NPB:HAT-CN bulk heterojunctions with various D-A blend ratios (solid lines). The HAT-CN concentration was varied from $4.5 \mathrm{wt} \%$ (20:1 blend) to 67 wt $\%$ (1:2 blend). The sky blue and maroon dashed lines are scaled EQE from single layers of NPB and HAT-CN, respectively. The red, green and blue colored boxes designate the three primary CT features in this system. The red dashed line is the absorptance of NPB:HAT-CN (20:1) film measured by photothermal deflection spectroscopy (PDS). 
The EQE spectra of NPB:HAT-CN BHJs change with the D:A mixing ratio, as shown in Fig. 2. As the HAT-CN concentration increases from $4.5 \mathrm{wt} \%$ (i.e. NPB rich) to $67 \mathrm{wt} \%$ (i.e. HAT-CN rich), an increase of D-A interfacial volume leads to an increase of overall CT state EQE, ${ }^{27,28}$ peaking for the 1:1 blend, while the three distinct $\mathrm{CT}$ features gradually merge. At the same time, $V_{\mathrm{OC}}$ of these devices decrease significantly (Fig. S2, Table S2) with increasing HAT$\mathrm{CN}$ concentration. The decrease of $V_{\mathrm{OC}}$ is due both to the increase in $\mathrm{CT}$ mediated recombination at the NPB:HAT-CN interface $\mathrm{e}^{23,27,28}$ and to the increasing population of NPB cations and HAT$\mathrm{CN}$ anions that are introduced by charge transfer (i.e. doping). Temperature dependent current density-voltage measurements (Fig. S3a) show that $V_{O C}$ increases continuously as temperature decreases, for both 20:1 and 1:2 NPB:HAT-CN blends, indicating the presence of strong recombination at room temperature. The higher dark current of the 1:2 NPB:HAT-CN device at small forward voltage (Fig. S3b) also indicates higher recombination as compared to those in the 20:1 NPB:HAT-CN device. The concomitant increase in recombination and sub-bandgap EQE, as the D-A blend goes from non-equimolar to equimolar ratios, provides further support that the sub-bandgap spectra of NPB:HAT-CN BHJs indeed originate from CT state absorption. ${ }^{28}$
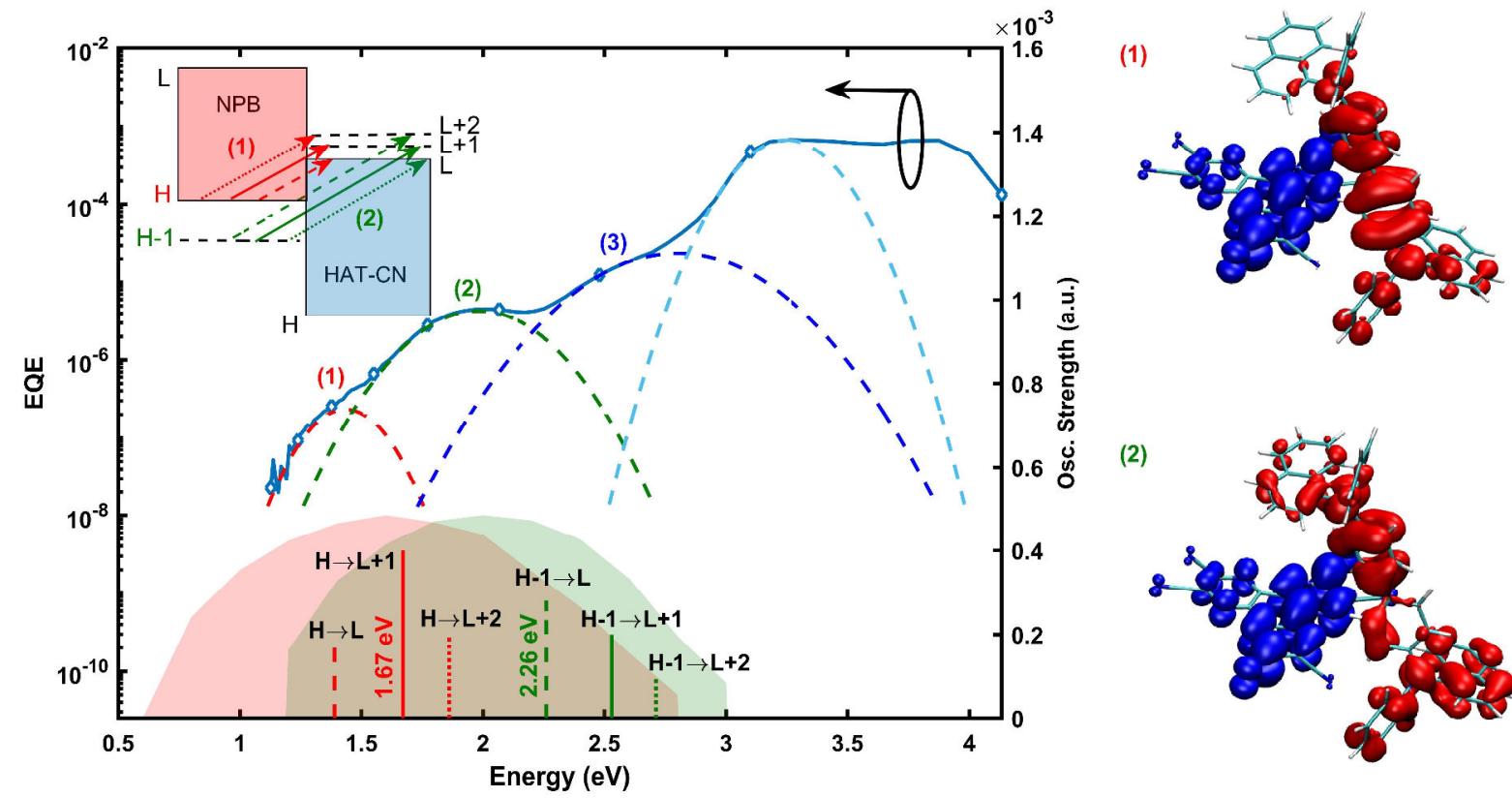

(2)

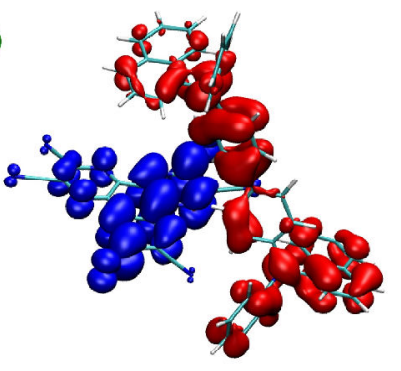

Figure 3. External quantum efficiency (EQE) spectra of a NPB:HAT-CN(20:1) (blue solid line) device. Four Gaussian functions fit to the $\mathrm{EQE}$ spectrum, yielding $\mathrm{CT}_{1}$ (dashed red line, center: $1.44 \mathrm{eV}, \mathrm{FWHM}$ : $0.31 \mathrm{eV}$ ), $\mathrm{CT}_{2}$ (dashed green line, center: $1.98 \mathrm{eV}, \mathrm{FWHM}$ : $0.50 \mathrm{eV}$ ), $\mathrm{CT}_{3}$ (dashed blue line, center: 2.78 eV, FWHM: $0.65 \mathrm{eV}$ ), and the NPB Frenkel exciton (dashed light blue line, center: $3.27 \mathrm{eV}$, FWHM: $0.37 \mathrm{eV}$ ). The red, green shaded Gaussians established from combining MD simulations and electronic structure calculations show the energetic distribution of $\mathrm{CT}_{1}$, and $\mathrm{CT}_{2}$ centered at $1.62 \mathrm{eV}$, and $2.01 \mathrm{eV}$, respectively. The red, and green delta lines represent CT energies calculated from TD-DFT of an NPBHAT-CN dimer, among which are two primary ones at $1.67 \mathrm{eV}$, and $2.26 \mathrm{eV}$, respectively, with their electron (blue) and hole (red) wavefunction distributions shown at the right. The inset displays transitions from NPB HOMO $(\mathrm{H})$, and HOMO-1 (H-1) to HAT-CN LUMOs (L, L+1 and L+2), which contribute 
mostly to the wavefunctions of the lowest $\mathrm{CT}$ states. The $\mathrm{CT}_{3}$ region represents higher energy $\mathrm{CT}$ states, associated with transitions from deeper HOMO levels of NPB to higher energy LUMO levels of HAT$\mathrm{CN}$, along with some contribution from NPB polaron (cation) absorption.

In order to shed light onto the different features appearing in the EQE spectrum of NPB:HAT-CN blends, we performed multiscale modeling analysis combining MD simulations and electronic structure calculations, capable of unraveling the complex interplay of conformational and environmental effects in disordered heterogeneous blends (see methods section and supplementary information for details). Our analysis reveals that these very broad CT features in the EQE spectra result from a combination of three different factors, namely: (i) the presence of three nearly degenerate acceptor levels for the HAT-CN molecule (at the crystalline geometry the LUMO, is two-fold degenerate and the LUMO+2 lies just $0.16 \mathrm{eV}$ above); (ii) the large fluctuation of NPB molecular energy levels due to conformational effects in the disordered structure; (iii) the variations in the local environment of the molecules resulting in a marked energetic disorder due to intermolecular electrostatic interactions.

For an easier comparison with theoretical data, in Fig. 3 we show multi-Gaussian fits to the EQE of an NPB:HAT-CN(20:1) device, yielding $\mathrm{CT}_{1}$ centered at $1.44 \mathrm{eV}$ with $0.31 \mathrm{eV}$ full width at half maximum (FWHM), $\mathrm{CT}_{2}$ at $1.98 \mathrm{eV}$ with $0.50 \mathrm{eV} \mathrm{FWHM}$, and $\mathrm{CT}_{3}$ at $2.78 \mathrm{eV}$ with 0.65 eV FWHM. Given that NPB:HAT-CN(20:1) films are amorphous, multiple CT features in the EQE are unlikely to originate from local morphological heterogeneities in nanocrystalline domains. ${ }^{9,18,19}$ Besides the experimental EQE, Fig. 3 shows, for an amorphous (13:1) simulated sample, the calculated density of states (DOS) for the two lowest-energy CT excitations (red shaded region for $\mathrm{CT}_{1}$ and green shaded region for $\mathrm{CT}_{2}$ ), which closely match the two lowest energy EQE features. The $\mathrm{CT}_{1}$ transitions correspond to transition from the NPB HOMO to the HAT-CN LUMOs, while $\mathrm{CT}_{2}$ relates to analogous excitations from the NPB HOMO-1 (see sketch in the figure inset). This analysis is further corroborated by time-dependent density functional theory (TD-DFT) calculations on an NPB-HAT-CN dimer extracted from the MD sample (vertical bars), which shows two series of absorption bands, $\mathrm{CT}_{1}$ (red) and $\mathrm{CT}_{2}$ (green), each of which consist of three transitions to the acceptor HAT-CN LUMO levels. The hole and electron densities of the $\mathrm{CT}_{1}$ and $\mathrm{CT}_{2}$ transitions to the HAT-CN LUMO are displayed in the right panels of Fig. 3. A precise assignment of the much broader band (3) in the EQE spectrum is less straightforward. In fact, we expect the occurrence of additional higher-energies CT states, corresponding to transitions from deeper levels of NPB or to higher energy unoccupied levels of HAT-CN. These higher-energy CT states are likely to hybridize with localized excitations (LE), which are also expected to produce broad sub-bandgap features at D-A heterojunctions. ${ }^{17,29}$ In addition, we cannot exclude a possible contribution from the NPB cation (polaron) around 2.6 $\mathrm{eV}$.

Our multiscale simulations rationalize also the possibility for a slight doping in NPB:HAT-CN. Doping is expected to occur when the energy of the adiabatic (i.e. at the relaxed molecular structures) CT states is lower or comparable to the energy of the neutral system. ${ }^{30}$ The low-energy tail of the calculated vertical $\mathrm{CT}_{1}$ DOS in Fig. 3 extends down to $\sim 0.6 \mathrm{eV}$. From 
these values, the energy of adiabatic CT states can be approximately obtained by subtracting the hole and electron relaxation energies of NPB $\left(\lambda^{+}=0.33 \mathrm{eV}\right)$ and HAT-CN $\left(\lambda^{-}=0.08 \mathrm{eV}\right)$ to the vertical (i.e. at frozen structure) energy. This simple argument yields adiabatic CT excitations at energies below $0.2 \mathrm{eV}$, hence suggesting the existence of a small fraction of ionized NPB molecules in the blend.
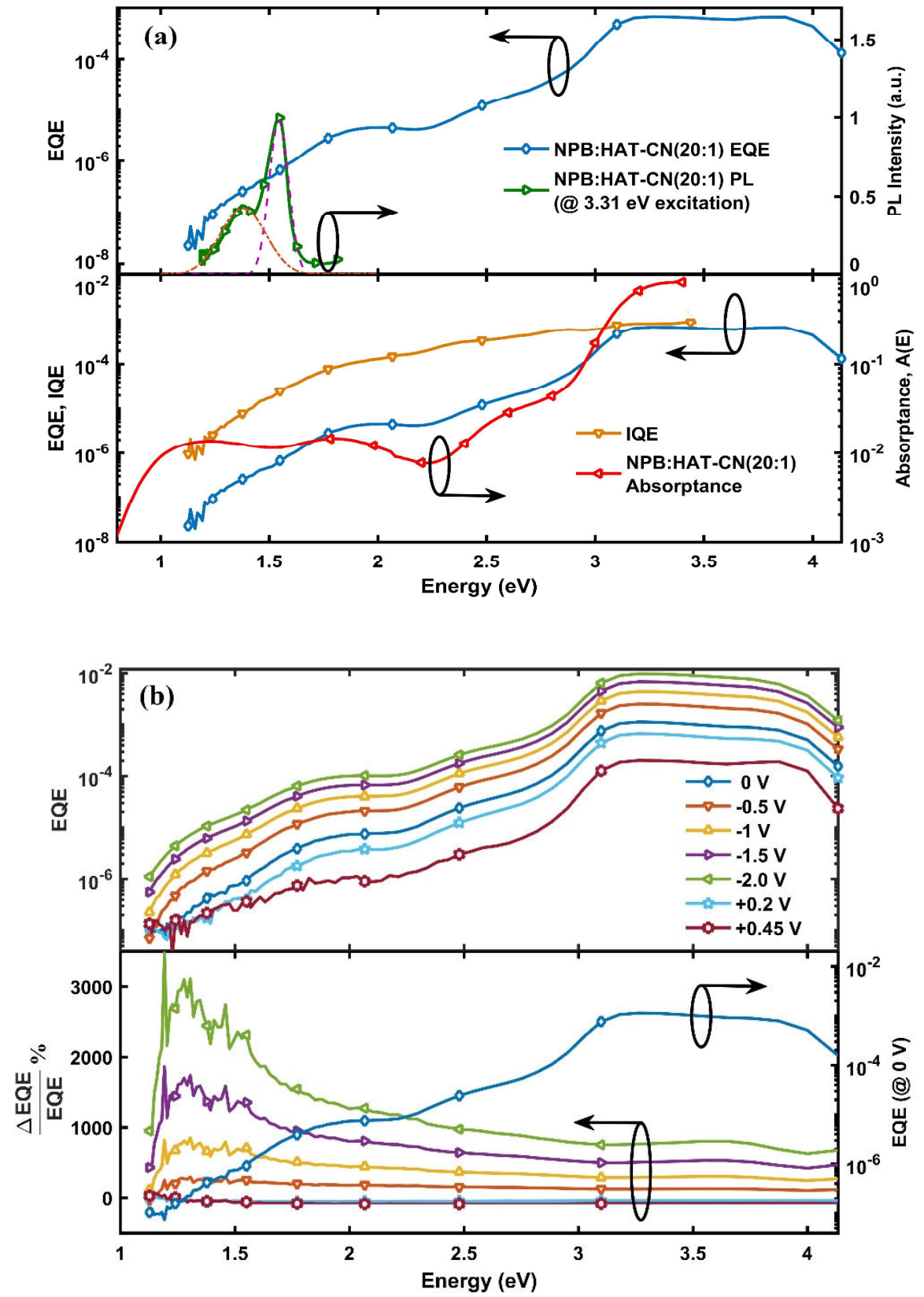

Figure 4. (a) [top] Photoluminescence spectrum of an NPB:HAT-CN(20:1) film excited at $3.31 \mathrm{eV}$ (green solid line). The purple and red dashed lines show two distinct peaks in the PL spectra, located at $1.55 \mathrm{eV}$ and $1.38 \mathrm{eV}$. [bottom] Absorptance of a 20:1 NPB:HAT-CN film measured via PDS (red). Estimated IQE (gold) and EQE spectra of an NPB:HAT-CN(20:1) device (blue). The IQE exhibits two order of magnitude drop from 2.0 to $1.1 \mathrm{eV}$. (b) [top] EQE of a NPB:HAT-CN(20:1) device at different voltage 
bias ranging from $-2 \mathrm{~V}$ to $+0.45 \mathrm{~V}$. [bottom] Percent change in EQE with applied bias, showing that the lowest energy CT state is most sensitive to applied reverse bias.

The presence of different electronic CT state features in the EQE provides us with a unique opportunity to study their role in photocurrent generation and recombination. To this end, Fig. 4a displays the PL spectrum from a NPB:HAT-CN(20:1) film excited at $3.31 \mathrm{eV}$ (wavelength $\lambda=375 \mathrm{~nm}$ ). The spectrum is fitted by two Gaussian functions centered at 1.55 and $1.38 \mathrm{eV}$, most likely corresponding to the broad $\mathrm{CT}_{2}$ feature at around $2.0 \mathrm{eV}$ in the EQE, considering that typically a reorganization energy of $0.4-0.6 \mathrm{eV}$ exists between absorption (EQE) and emission (PL). Surprisingly, no PL is observed for CT features (1) and (3) (cf. Fig. 3). Additionally, no PL signals are observed when exciting the film directly into the CT manifold, at $1.94 \mathrm{eV}(\lambda=638 \mathrm{~nm})$, most likely due to the lack of excited state population manifested by the weak absorption (negligible transition dipole moment) between the ground and CT states.

The bottom panel of Fig. 4a shows the internal quantum efficiency (IQE) of a 20:1 NPB:HAT-CN blend device calculated from a transfer matrix model. ${ }^{31,32}$ We find that IQE is relatively constant in the Frenkel and high energy CT state absorption regions, but that it drops off sharply at energies below $2.3 \mathrm{eV}$. This suggests inequivalent dissociation and recombination rates among the different $\mathrm{CT}$ states in the manifold. Examining EQE with applied voltage bias in Fig. 4b shows that the lowest energy CT state experiences the largest change with applied reverse bias, indicating that polaron pairs relaxed to the lowest energy CT states do not contribute to photocurrent generation effectively and instead act as a major recombination pathway. This therefore provides an additional, ${ }^{9}$ but not necessarily generalizable, counterexample to the mainstream idea that relaxed CT states contribute equally to photocurrent compared to localized Frenkel excitations or other, higher energy, CT states. ${ }^{10}$ 


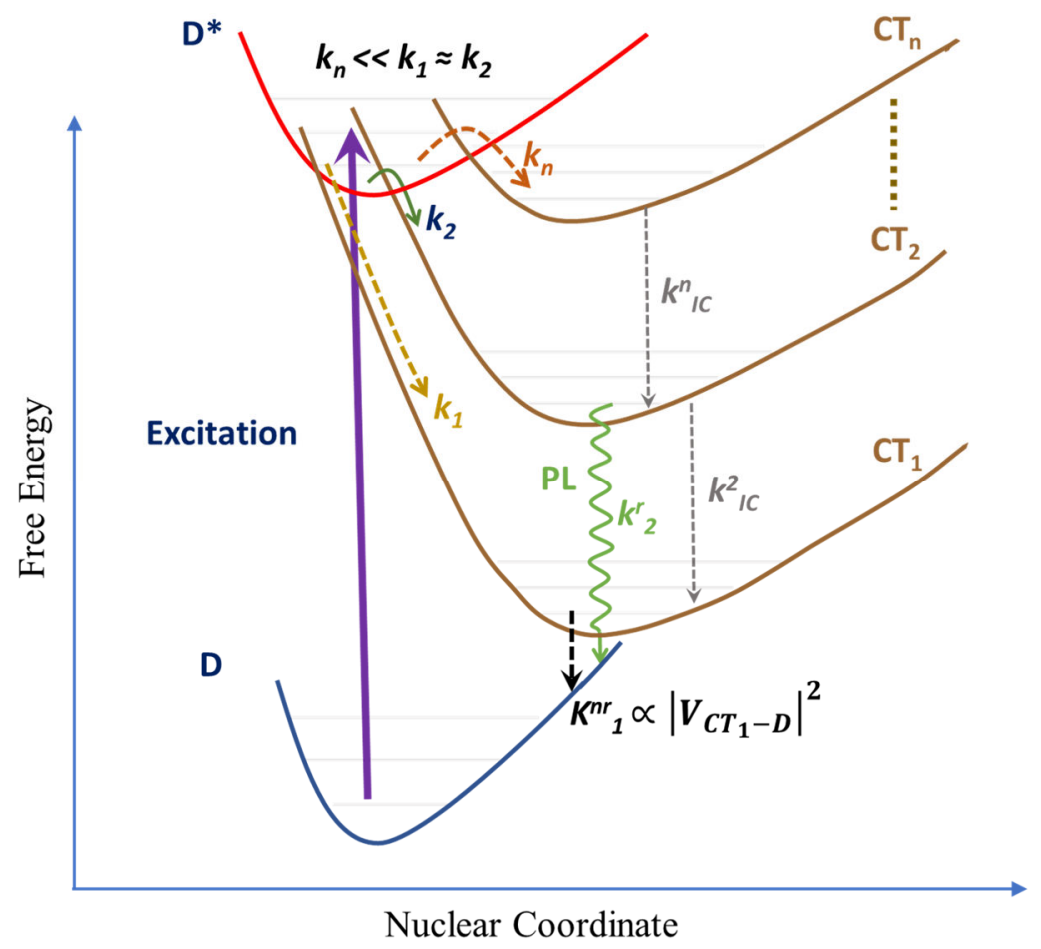

Figure 5. Proposed state diagram of the $\mathrm{CT}_{1}, \mathrm{CT}_{2}$ and higher energy $\mathrm{CT}_{\mathrm{n}}$ electronic levels, along with the ground (D) and excited state (D*) energy levels. Upon excitation of the donor, PL is observed only from $\mathrm{CT}_{2}$ at a rate $k_{2}^{r}$. This could be explained by assuming that $\mathrm{CT}_{1}$ is in the Marcus inverted region and $\mathrm{CT}_{\mathrm{n}}$ has a large barrier, thus only $\mathrm{CT}_{2}$ can be populated from a localized intramolecular Frenkel excitation. $k_{l}$, $k_{2}$ and $k_{n}$ are charge transfer rates from $\mathrm{D}^{*}$ to $\mathrm{CT}_{1}, \mathrm{CT}_{2}$ and $\mathrm{CT}_{\mathrm{n}}$ respectively, where $k_{n}<<k_{1} \approx k_{2} . k^{n}$ IC the internal conversion rate from $\mathrm{CT}_{\mathrm{n}}$ to $\mathrm{CT}_{\mathrm{n}-1}$ state. $k^{2}{ }_{I C}$ has to be smaller than $k_{2}^{r}$, to sustain PL emission from $\mathrm{CT}_{2} . k^{n r}{ }_{1}$ is the non-radiative recombination rate from the lowest energy $\mathrm{CT}$ state $\left(\mathrm{CT}_{1}\right)$ to ground states (D), which is proportional to the $\mathrm{CT}_{1}-\mathrm{D}$ state coupling, $V_{C T I-D}$.

Finally, we propose the state diagram shown in Fig. 5 to explain our collective observations of the NPB:HAT-CN system. The large D-A energy offset leads to a broad subbandgap distribution of CT states, which increases the chance, or perhaps even ensures, that the lowest energy CT state falls in the Marcus inverted region and experiences an elevated activation barrier during the transition from Frenkel $\left(D^{*}\right)$ to CT state. High energy CT states (i.e. $\mathrm{CT}_{2}, \ldots$, $\mathrm{CT}_{\mathrm{n}}$, where $\left.\mathrm{n}=3,4, \ldots\right)$ in the normal Marcus region follow the trend that the activation barrier increases with the CT state energy. Charge transfer rate from $\mathrm{D}^{*}$ to $\mathrm{CT}_{1}, \mathrm{CT}_{2}$ and $\mathrm{CT}_{\mathrm{n}}$, therefore, follow the relationship that $k_{n}<<k_{1} \approx k_{2}$, where the subscript numbers correspond to the CT state electronic levels. As shown in Fig. 3, $\mathrm{CT}_{1}$ and $\mathrm{CT}_{2}$ are primarily composed of NPB HOMO to HAT-CN LUMO+1, and NPB HOMO-1 to HAT-CN LUMO transitions, respectively. Internal conversion from $\mathrm{CT}_{2}$ to $\mathrm{CT}_{1}$ would require that electrons in HAT-CN LUMO spontaneously upconvert to LUMO+1 and holes in NPB relax from HOMO-1 to HOMO (see Fig. S5 for the energy levels of NPB and HAT-CN frontier orbitals). The up-conversion rate of the electron and relaxation rate of the holes are limited by the availability of excess thermal energy and the 
wavefunction overlap of HOMO-1 and HOMO in NPB (shown in red, at the right of Fig. 3), respectively. Overall, these ensure that once $\mathrm{CT}_{2}$ excitons are formed, the internal conversion from $\mathrm{CT}_{2}$ to $\mathrm{CT}_{1}$ (with rate $k_{I C}^{2}$ ) competes with the radiative recombination (at rate $k_{2}^{r}$ ) from $\mathrm{CT}_{2}$ to $\mathrm{D}$ (i.e. $k_{2}^{r}>k_{I C}^{2}$ ) and PL from $\mathrm{CT}_{2}$ is observed. At the same time, the small $\mathrm{CT}$ state energy and strong electronic coupling of $\mathrm{CT}_{1}$ to the ground state, result in dominant non-radiative recombination (at rate $\left.k_{1}^{n r}\right)^{33}$ from $\mathrm{CT}_{1}$ to $\mathrm{D}$, following the usual energy gap law of organic molecules, ${ }^{34}$ and no PL emission is observed from this state.

\section{Conclusions}

We have demonstrated several organic D-A interfaces with unconventionally broad CT state features $(>2 \mathrm{eV})$ and performed an in-depth study on NPB-HAT-CN BHJs. The broad CT features in NPB-HAT-CN system were determined to originate from multiple electronic CT state energies broadened by conformational and dielectric environment heterogeneities. From a device performance standpoint, these devices are intendedly far from ideal, as they are incapable of realizing large photocurrent (due to the high donor and acceptor optical gap energies) and $V_{\mathrm{OC}}$ (due to very large orbital offset energies and strong non-radiative recombination). However, this heterojunction energetic design has allowed us to explore CT state spectral features over a broad range of photon energies, to probe their properties in ways not feasible in other D-A systems, and to better learn about the role of CT states in energy loss and charge generation. In the NPB:HAT$\mathrm{CN}$ example that we studied in detail, ultrasensitive absorption measurement revealed low energy CT states, which are not evident from EQE measurements. The estimated IQE of the device, along with the voltage bias dependent EQE, revealed that carrier dissociation efficiency in these devices is strongly photon energy dependent and that the low energy CT state suffers from severe recombination, owing to strong electronic coupling to the ground state.

\section{Acknowledgments}

This work was supported in part by the U.S. Department of Energy, Office of Basic Energy Sciences under Award Nos. DE-SC0012365 and DE-SC0012458, by the European Union's Horizon 2020 research and innovation program under Grant Agreement No. 646176 (EXTMOS project) and by European Union's Horizon 2020 research and innovation program under the Marie Skłodowska-Curie Grant agreement No. 722651 (SEPOMO project). Computational resources were provided by the Consortium des Équipements de Calcul Intensif (CÉCI), funded by the Fonds de la Recherche Scientifiques de Belgique (F.R.S.-FNRS) under Grant No. 2.5020.11, as well as the Tier-1 supercomputer of the Fédération Wallonie-Bruxelles, infrastructure funded by the Walloon Region under Grant Agreement No. 1117545. D.B. is a FNRS Research Director. T.C.-J.Y acknowledges the support of a Marie Skłodowska-Curie Individual Fellowship from the European Union's Horizon 2020 research and innovation program (grant agreement No. 747221, action acronym: POSITS). 


\section{Methods}

Device and film preparation: All materials were purchased from commercial vendors; Bathocuproine (BCP) (Lumtec), NPB (Nichem), m-TDATA (Sigma Aldrich), NTCDA (TCI America), TAPC (Lumtec), HAT-CN (Nichem), and PVK (Sigma Aldrich). NPB, m-TDATA, NTCDA were purified via thermal gradient sublimation prior to use whereas other materials were used as received.

Pre-patterned ITO glass substrates (Colorado Concept Coatings) were used for solar cells. Quartz (AdValue Technology) substrates were used for absorption and photoluminescence measurements. All substrates were cleaned by successive sonication in deionized water $\left(40{ }^{\circ} \mathrm{C}\right.$, $15 \mathrm{~min}$ ), acetone $\left(40{ }^{\circ} \mathrm{C}, 10 \mathrm{~min}\right)$ and isopropanol $\left(40^{\circ} \mathrm{C}, 10 \mathrm{~min}\right)$, followed by oxygen plasma treatment for $10 \mathrm{~min}$.

The small molecule devices were fabricated using thermal evaporation with $\sim 10^{-7}$ torr base pressure. The basic structure of the devices were ITO/donor:acceptor/BCP $(8-10 \mathrm{~nm}) / \mathrm{Al}$ $(100 \mathrm{~nm})$. All active layers of the devices and the films ranged from $40 \mathrm{~nm}$ to $150 \mathrm{~nm}$ in thickness. The small molecule organic donor materials were co-evaporated with the acceptor materials, to make a bulk heterojunction (BHJ). Since the acceptor NTCDA tends to crystallize, ${ }^{35}$ its weight fraction was kept small to ensure a disordered film morphology. As for HAT-CN, although the vacuum deposited films do not suffer from crystallization, ${ }^{36}$ the weight ratio was kept relatively low to avoid any aggregation or crystallization induced effects. We have fabricated BHJ devices with NPB:HAT-CN (20:1), NPB:NTCDA (10:1), TAPC:HAT-CN (20:1), m-TDATA:NTCDA (10:1) bulk active layers, where the blend ratio denotes mixing of the donor and acceptor materials by weight.

For the polymer donor PVK, planar heterojunction (PHJ) devices were formed with HAT-CN by solution processing, due to the lack of a suitable common solvent for PVK and HAT-CN. We have deposited HAT-CN by both solution processing and vacuum deposition, with both devices producing the same broad sub-bandgap EQE spectrum (Fig. S4). To make devices, PEDOT:PSS (Heraeus) was spin-coated on cleaned ITO-glass substrates at $5000 \mathrm{rpm}$ for $60 \mathrm{~s}$, followed by a $20 \mathrm{~min}$ annealing at $140{ }^{\circ} \mathrm{C}$. PVK films were formed by spin coating $5 \mathrm{mg} / \mathrm{ml}$ solution (in chlorobenzene (Sigma Aldrich)) at $1000 \mathrm{rpm}$ for $60 \mathrm{~s}$. HAT-CN layer was formed by spin coating from a $5 \mathrm{mg} / \mathrm{ml}$ solution as well (in acetonitrile (Sigma Aldrich)), at $2000 \mathrm{rpm}$ for $30 \mathrm{~s}$. Films were annealed at $150{ }^{\circ} \mathrm{C}$, for $20 \mathrm{~min}$. The entire active layer preparation was done in a $\mathrm{N}_{2}$ filled glovebox and later transferred to a thermal deposition chamber for depositing $\mathrm{BCP}$ and $\mathrm{Al}$ contact on top of the films. The active area of all devices was $0.1 \mathrm{~cm}^{2}$.

IV and EQE Characterization: The current-voltage characteristics of the solar cells were measured using a solar simulator (ABET Technologies) calibrated for 1 sun AM1.5G illumination and a Keithley 2400 source meter. The EQE measurements were taken using a Newport TLS-300X tunable light source at short-circuit condition unless otherwise noted. The monochromatic light output from the light source was chopped at $390 \mathrm{~Hz}$ using an optical chopper. Device photocurrent was amplified by a current pre-amplifier (SR570, Stanford Research Systems) and detected by a lock-in amplifier (SR830, Stanford Research Systems) 
tuned to the optical chopper frequency. To count the number of incoming photons from the light source, calibrated Si and Ge photodiodes from Newport were used as reference cells.

EQE Under Voltage Bias: External voltage bias was applied to the solar cell using a current preamplifier (SR570). To avoid signal overload at the pre-amplifier output, the applied voltage was kept under $-2 \mathrm{~V}$ reverse and $+0.45 \mathrm{~V}$ forward bias. The pre-amplifier was operated at low noise mode and a $300 \mathrm{~Hz}-1 \mathrm{kHz}$ bandpass filter was applied to the incoming signal. The pre-amplifier gain was set to $100 \mathrm{nA} / \mathrm{V}$ throughout the EQE measurement.

Low Temperature Voc Measurement: Low temperature $V_{\mathrm{OC}}$ was measured by placing the device inside a Janis VNF-100 Cryostat. The temperature of the device was monitored and controlled by a LakeShore 335 Cryogenic Temperature Controller. A Newport 250-Watt QTH lamp (Newport) was calibrated to 1 sun relative intensity to illuminate the device and the current-voltage characteristics were measured using a Keithley 2400 source meter. Measurements were done by first cooling down the sample to $80 \mathrm{~K}$ and then gradually heating up to the room temperature. No sign of device degradation was observed due to this cool down and heating up process.

Photoluminescence Measurement: Photoluinescence (PL) was collected from a NPB:HAT-CN blend film excited using a continuous-wave, $\lambda=375 \mathrm{~nm}(3.31 \mathrm{eV})$ excitation laser and a Horiba iHR fibre-coupled spectrometer with a cooled Si CCD array detector.

Photothermal Deflection Spectroscopy: Photothermal deflection spectroscopy (PDS) measurements were taken using a $150 \mathrm{~W}$ Xenon lamp equipped with a three-grating monochromator. Samples were immersed in Fluorinert FC-72 $\left(\mathrm{C}_{6} \mathrm{~F}_{14}\right)$, a liquid with a strongly temperature-dependent refractive index, which equals 1.25 at room temperature. Absorption is measured based on the deflection of a laser beam (JDSU 1137P, $7.0 \mathrm{~mW}, 632.8 \mathrm{~nm}$ NeonHelium, linear polarized) by thermally induced changes in the refractive index of the liquid in which the illuminated sample is immersed. The PDS spectra were calibrated to absolute absorptance scale by matching with the UV-Vis absorption spectra at $3.18 \mathrm{eV}$, measured by a PerkinElmer 950 spectrophotometer using an integrating sphere.

IQE Calculation: The absorption $a(E)$ of D:A blend in a full device comprising glass/ITO/D:A/BCP/Al was calculated using a transfer matrix model ${ }^{31}$ where parasitic absorption and optical interference effects are considered. The light was incident normal to the substrate via the ITO anode side. The optical constants $(n, k)$ and thickness of each layer in the model were all measured by ellipsometry, except that $k$ of D:A layer was obtained from PDS absorptance data, ensuring clearly resolved CT state features. The IQE was then given by EQE/( $a(E))$.

Molecular Dynamics Simulations: A film of 9:1 NPB:HAT-CN m/m ratio (about 13:1 in weight) was prepared by vapor co-deposition on graphene by means of NVT non-equilibrium molecular dynamics (MD) simulations ${ }^{37,38}$ for a final thickness exceeding $100 \AA$. The system was modelled with a previously developed united atom force field. ${ }^{39}$ United atom charges were obtained by fitting the electrostatic potential at the optimized geometry calculated at the DFT PBE0 $40 /$ def2- 
TZVP level of theory. A horizontal graphene surface of $76.14 \AA \times 76.57 \AA$ was chosen as a simple rigid support and kept fixed during the simulations, with a vertical box dimension of 400 $\AA$. Deposition proceeded by randomly selecting the chemical species of the new molecule to be added at each step, according to the desired molar ratio. Every new molecule was placed at about $20 \AA$ above the forming organic layer, endowed with a velocity of $1 \AA \mathrm{ps}^{-1}$ directed towards the graphene surface, then the dynamic of the system evolved for 100 ps at $500 \mathrm{~K}$. In the case of desorption, the step was repeated without randomizing again the molecular species. The final composition of the sample was of 897 NPB and 103 HAT-CN molecules. The adopted scheme yielded an amorphous sample, as verified by inspecting radial distributions, linear mass density along the box sides and orientational order parameters. The system was subsequently equilibrated at $300 \mathrm{~K}$ for at least $60 \mathrm{~ns}$, and the final configuration was used to extract the molecular geometries for the calculations of electronic properties.

Electronic Structure Calculations: The DOS of CT states in Fig. 3 were calculated upon sampling over molecular conformations obtained from MD simulations. CT states energies were computed for all the NPB:HAT-CN dimers of the inner, bulk-like, region of the MD-deposited film as:

$$
E_{C T}=E_{A}-E_{D}+\Delta^{ \pm}
$$

where $E_{A}=E_{L+i}$ and $E_{D}=E_{H-j}$ are the gas-phase energies of the HAT-CN LUMO $+i(i=0,1,2)$ and of the NPB HOMO- $j(j=0,1)$ orbitals, respectively. $\Delta^{ \pm}$is the ion pair polarization energies that accounts for the electron-hole Coulomb attraction and environmental effects in the disordered blend. The latter was computed from self-consistent MicroElectrostatic calculations as implemented in the MESCAL code. ${ }^{41}$

Gas-phase energy levels were calculated combining evGW calculations, ensuring very accurate absolute values, and DFT, allowing the sampling of molecular conformations and of the corresponding energy level fluctuations. The energy of a generic energy level $k$ reads:

$$
E_{k}\left(r_{M D}\right)=E_{k}^{G W}\left(r_{0}\right)+\left[E_{k}^{D F T}\left(r_{M D}\right)-E_{k}^{D F T}\left(r_{0}\right)\right]
$$

where $r_{M D}$ labels the geometry of a given molecule in the MD sample and $r_{0}$ is a reference geometry, taken from the bulk crystal structure of both species. ev $G W$ calculations (PBE0 $0^{40}$ functional, complete basis set limit) calculations were performed with the Fiesta code. ${ }^{42,43}$ DFT energy levels and relaxation energies were computed at the $\omega \mathrm{B} 97 \mathrm{X}-\mathrm{D}^{44} / 6-311++\mathrm{G}(\mathrm{d}, \mathrm{p})$ level.

TD-DFT calculations were performed for NPB:HAT-CN dimers extracted from the MD sample at the CAM-B3LYP ${ }^{45} / 6-31 \mathrm{G}(\mathrm{d}, \mathrm{p})$ level, accounting for a continuum polarizable ${ }^{46}$ embedding with dielectric constant $\varepsilon_{r}=3$. DFT and TD-DFT calculations were carried out with the GAUSSIAN $16^{47}$ suite. 


\section{References}

(1) Green, M. A.; Hishikawa, Y.; Dunlop, E. D.; Levi, D. H.; Hohl-Ebinger, J.; Yoshita, M.; Ho-Baillie, A. W. Y. Solar Cell Efficiency Tables (Version 53). Prog. Photovoltaics Res. Appl. 2019, 27 (1), 3-12. https://doi.org/10.1002/pip.3102.

(2) Gao, F.; Inganäs, O. Charge Generation in Polymer-Fullerene Bulk-Heterojunction Solar Cells. Phys. Chem. Chem. Phys. 2014. https://doi.org/10.1039/c4cp01814a.

(3) Clarke, T. M.; Durrant, J. R. Charge Photogeneration in Organic Solar Cells. Chem. Rev. 2010. https://doi.org/10.1021/cr900271s.

(4) Vandewal, K.; Tvingstedt, K.; Gadisa, A.; Inganäs, O.; Manca, J. V. On the Origin of the Open-Circuit Voltage of Polymer-Fullerene Solar Cells. Nat. Mater. 2009. https://doi.org/10.1038/nmat2548.

(5) Linderl, T.; Zechel, T.; Brendel, M.; Moseguí González, D.; Müller-Buschbaum, P.; Pflaum, J.; Brütting, W. Energy Losses in Small-Molecule Organic Photovoltaics. Adv. Energy Mater. 2017. https://doi.org/10.1002/aenm.201700237.

(6) Burke, T. M.; Sweetnam, S.; Vandewal, K.; McGehee, M. D. Beyond Langevin Recombination: How Equilibrium between Free Carriers and Charge Transfer States Determines the Open-Circuit Voltage of Organic Solar Cells. Adv. Energy Mater. 2015. https://doi.org/10.1002/aenm.201500123.

(7) Grancini, G.; Maiuri, M.; Fazzi, D.; Petrozza, A.; Egelhaaf, H. J.; Brida, D.; Cerullo, G.; Lanzani, G. Hot Exciton Dissociation in Polymer Solar Cells. Nat. Mater. 2013. https://doi.org/10.1038/nmat3502.

(8) Ohkita, H.; Cook, S.; Astuti, Y.; Duffy, W.; Tierney, S.; Zhang, W.; Heeney, M.; McCulloch, I.; Nelson, J.; Bradley, D. D. C.; et al. Charge Carrier Formation in Polythiophene/Fullerene Blend Films Studied by Transient Absorption Spectroscopy. J. Am. Chem. Soc. 2008, 130 (10), 3030-3042. https://doi.org/10.1021/ja076568q.

(9) Liu, X.; Ding, K.; Panda, A.; Forrest, S. R. Charge Transfer States in Dilute DonorAcceptor Blend Organic Heterojunctions. ACS Nano 2016. https://doi.org/10.1021/acsnano.6b02865.

(10) Vandewal, K.; Albrecht, S.; Hoke, E. T.; Graham, K. R.; Widmer, J.; Douglas, J. D.; Schubert, M.; Mateker, W. R.; Bloking, J. T.; Burkhard, G. F.; et al. Efficient Charge Generation by Relaxed Charge-Transfer States at Organic Interfaces. Nat. Mater. 2014. https://doi.org/10.1038/nmat3807.

(11) Lee, J.; Vandewal, K.; Yost, S. R.; Bahlke, M. E.; Goris, L.; Baldo, M. A.; Manca, J. V.; Voorhis, T. Van. Charge Transfer State versus Hot Exciton Dissociation in PolymerFullerene Blended Solar Cells. J. Am. Chem. Soc. 2010. https://doi.org/10.1021/ja1045742.

(12) Sánchez-Carrera, R. S.; Paramonov, P.; Day, G. M.; Coropceanu, V.; Brédas, J. L. Interaction of Charge Carriers with Lattice Vibrations in Oligoacene Crystals from Naphthalene to Pentacene. J. Am. Chem. Soc. 2010. https://doi.org/10.1021/ja1040732.

(13) Tummala, N. R.; Zheng, Z.; Aziz, S. G.; Coropceanu, V.; Brédas, J.-L. Static and Dynamic Energetic Disorders in the C60, PC61BM, C70, and PC71BM Fullerenes. $J$. Phys. Chem. Lett. 2015. https://doi.org/10.1021/acs.jpclett.5b01709.

(14) Unger, T.; Wedler, S.; Kahle, F. J.; Scherf, U.; Bässler, H.; Köhler, A. The Impact of Driving Force and Temperature on the Electron Transfer in Donor-Acceptor Blend 
Systems. J. Phys. Chem. C 2017. https://doi.org/10.1021/acs.jpcc.7b09213.

(15) Tummala, N. R.; Elroby, S. A.; Aziz, S. G.; Risko, C.; Coropceanu, V.; Brédas, J. L. Packing and Disorder in Substituted Fullerenes. J. Phys. Chem. C 2016. https://doi.org/10.1021/acs.jpcc.6b05197.

(16) Belova, V.; Beyer, P.; Meister, E.; Linderl, T.; Halbich, M. U.; Gerhard, M.; Schmidt, S.; Zechel, T.; Meisel, T.; Generalov, A. V.; et al. Evidence for Anisotropic Electronic Coupling of Charge Transfer States in Weakly Interacting Organic Semiconductor Mixtures. J. Am. Chem. Soc. 2017. https://doi.org/10.1021/jacs.7b01622.

(17) Chen, X.-K.; Coropceanu, V.; Brédas, J.-L. Assessing the Nature of the Charge-Transfer Electronic States in Organic Solar Cells. Nat. Commun. 2018, 9 (1), 5295. https://doi.org/10.1038/s41467-018-07707-8.

(18) Lin, Y. L.; Zhang, F.; Kerner, R. A.; Yang, T. C.-J.; Kahn, A.; Rand, B. P. Variable Charge Transfer State Energies at Nanostructured Pentacene/C 60 Interfaces. Appl. Phys. Lett. 2018, 112 (21), 213302. https://doi.org/10.1063/1.5030885.

(19) Lin, Y. L.; Fusella, M. A.; Rand, B. P. The Impact of Local Morphology on Organic Donor/Acceptor Charge Transfer States. Adv. Energy Mater. 2018, 8 (28), 1702816. https://doi.org/10.1002/aenm.201702816.

(20) Ding, K.; Liu, X.; Forrest, S. R. Charge Transfer and Collection in Dilute Organic DonorAcceptor Heterojunction Blends. Nano Lett. 2018, 18 (5), 3180-3184. https://doi.org/10.1021/acs.nanolett.8b00851.

(21) Roland, S.; Kniepert, J.; Love, J. A.; Negi, V.; Liu, F.; Bobbert, P.; Melianas, A.; Kemerink, M.; Hofacker, A.; Neher, D. Equilibrated Charge Carrier Populations Govern Steady-State Nongeminate Recombination in Disordered Organic Solar Cells. J. Phys. Chem. Lett. 2019, 1374-1381. https://doi.org/10.1021/acs.jpclett.9b00516.

(22) Rau, U. Reciprocity Relation between Photovoltaic Quantum Efficiency and Electroluminescent Emission of Solar Cells. Phys. Rev. B - Condens. Matter Mater. Phys. 2007. https://doi.org/10.1103/PhysRevB.76.085303.

(23) Vandewal, K.; Tvingstedt, K.; Gadisa, A.; Inganäs, O.; Manca, J. V. Relating the OpenCircuit Voltage to Interface Molecular Properties of Donor:Acceptor Bulk Heterojunction Solar Cells. Phys. Rev. B - Condens. Matter Mater. Phys. 2010. https://doi.org/10.1103/PhysRevB.81.125204.

(24) Brigeman, A. N.; Fusella, M. A.; Rand, B. P.; Giebink, N. C. Nonthermal Site Occupation at the Donor-Acceptor Interface of Organic Solar Cells. Phys. Rev. Appl. 2018. https://doi.org/10.1103/PhysRevApplied.10.034034.

(25) Ma, H.; Troisi, A. Direct Optical Generation of Long-Range Charge-Transfer States in Organic Photovoltaics. Adv. Mater. 2014. https://doi.org/10.1002/adma.201402294.

(26) Brigeman, A. N.; Fusella, M. A.; Yan, Y.; Purdum, G. E.; Loo, Y. L.; Rand, B. P.; Giebink, N. C. Revealing the Full Charge Transfer State Absorption Spectrum of Organic Solar Cells. Adv. Energy Mater. 2016. https://doi.org/10.1002/aenm.201601001.

(27) Buchaca-Domingo, E.; Vandewal, K.; Fei, Z.; Watkins, S. E.; Scholes, F. H.; Bannock, J. H.; De Mello, J. C.; Richter, L. J.; DeLongchamp, D. M.; Amassian, A.; et al. Direct Correlation of Charge Transfer Absorption with Molecular Donor:Acceptor Interfacial Area via Photothermal Deflection Spectroscopy. J. Am. Chem. Soc. 2015. https://doi.org/10.1021/ja512410f.

(28) Vandewal, K.; Widmer, J.; Heumueller, T.; Brabec, C. J.; McGehee, M. D.; Leo, K.; Riede, M.; Salleo, A. Increased Open-Circuit Voltage of Organic Solar Cells by Reduced 
Donor-Acceptor Interface Area. Adv. Mater. 2014.

https://doi.org/10.1002/adma.201400114.

(29) D’Avino, G.; Muccioli, L.; Olivier, Y.; Beljonne, D. Charge Separation and

Recombination at Polymer-Fullerene Heterojunctions: Delocalization and Hybridization Effects. J. Phys. Chem. Lett. 2016, 7 (3), 536-540.

https://doi.org/10.1021/acs.jpclett.5b02680.

(30) Li, J.; D’Avino, G.; Pershin, A.; Jacquemin, D.; Duchemin, I.; Beljonne, D.; Blase, X. Correlated Electron-Hole Mechanism for Molecular Doping in Organic Semiconductors. Phys. Rev. Mater. 2017, 1 (2), 025602.

https://doi.org/10.1103/PhysRevMaterials.1.025602.

(31) Peumans, P.; Yakimov, A.; Forrest, S. R. Small Molecular Weight Organic Thin-Film Photodetectors and Solar Cells. J. Appl. Phys. 2003, 93 (7), 3693-3723.

https://doi.org/10.1063/1.1534621.

(32) Armin, A.; Velusamy, M.; Wolfer, P.; Zhang, Y.; Burn, P. L.; Meredith, P.; Pivrikas, A. Quantum Efficiency of Organic Solar Cells: Electro-Optical Cavity Considerations. $A C S$ Photonics 2014. https://doi.org/10.1021/ph400044k.

(33) Chen, X.-K.; Ravva, M. K.; Li, H.; Ryno, S. M.; Brédas, J.-L. Effect of Molecular Packing and Charge Delocalization on the Nonradiative Recombination of ChargeTransfer States in Organic Solar Cells. Adv. Energy Mater. 2016, 6 (24), 1601325. https://doi.org/10.1002/aenm.201601325.

(34) Köhler, A.; Bässler, H. Electronic Processes in Organic Semiconductors: An Introduction; 2015. https://doi.org/10.1002/9783527685172.

(35) Blackburn, A. C.; Fitzgerald, L. J.; Gerkin, R. E. 1,4,5,8-Naphthalenetetracarboxylic Acid Cyclic 1,8-Anhydride Bis(Dimethyl Sulfoxide) Solvate and 1,4,5,8-

Naphthalenetetracarboxylic 1,8:4,5-Dianhydride. Acta Crystallogr. Sect. C Cryst. Struct. Commun. 1997, 53 (12), 1991-1995. https://doi.org/10.1107/S0108270197011712.

(36) Wang, B.; Zhang, L.; Hu, Y.; Shi, X.-B.; Wang, Z.-K.; Liao, L.-S. Doped Hole Injection Bilayers for Solution Processable Blue Phosphorescent Organic Light-Emitting Diodes. J. Mater. Chem. C 2016, 4 (27), 6570-6574. https://doi.org/10.1039/C6TC01624C.

(37) D’Avino, G.; Muccioli, L.; Zannoni, C. From Chiral Islands to Smectic Layers: A Computational Journey Across Sexithiophene Morphologies on C 60. Adv. Funct. Mater. 2015, 25 (13), 1985-1995. https://doi.org/10.1002/adfm.201402609.

(38) Roscioni, O. M.; D’Avino, G.; Muccioli, L.; Zannoni, C. Pentacene Crystal Growth on Silica and Layer-Dependent Step-Edge Barrier from Atomistic Simulations. J. Phys. Chem. Lett. 2018, 9 (23), 6900-6906. https://doi.org/10.1021/acs.jpclett.8b03063.

(39) Moral, M.; Son, W.-J.; Sancho-García, J. C.; Olivier, Y.; Muccioli, L. Cost-Effective Force Field Tailored for Solid-Phase Simulations of OLED Materials. J. Chem. Theory Comput. 2015, 11 (7), 3383-3392. https://doi.org/10.1021/acs.jctc.5b00164.

(40) Adamo, C.; Barone, V. Toward Reliable Density Functional Methods without Adjustable Parameters: The PBE0 Model. J. Chem. Phys. 1999, 110 (13), 6158-6170. https://doi.org/10.1063/1.478522.

(41) D’Avino, G.; Muccioli, L.; Zannoni, C.; Beljonne, D.; Soos, Z. G. Electronic Polarization in Organic Crystals: A Comparative Study of Induced Dipoles and Intramolecular Charge Redistribution Schemes. J. Chem. Theory Comput. 2014, 10 (11), 4959-4971. https://doi.org/10.1021/ct500618w.

(42) Blase, X.; Attaccalite, C.; Olevano, V. First-Principles GW Calculations for Fullerenes, 
Porphyrins, Phtalocyanine, and Other Molecules of Interest for Organic Photovoltaic Applications. Phys. Rev. B 2011, 83 (11), 115103.

https://doi.org/10.1103/PhysRevB.83.115103.

(43) Li, J.; Duchemin, I.; Roscioni, O. M.; Friederich, P.; Anderson, M.; Da Como, E.; KociokKöhn, G.; Wenzel, W.; Zannoni, C.; Beljonne, D.; et al. Host Dependence of the Electron Affinity of Molecular Dopants. Mater. Horizons 2019, 6 (1), 107-114. https://doi.org/10.1039/C8MH00921J.

(44) Chai, J.-D.; Head-Gordon, M. Long-Range Corrected Hybrid Density Functionals with Damped Atom-atom Dispersion Corrections. Phys. Chem. Chem. Phys. 2008, 10 (44), 6615. https://doi.org/10.1039/b810189b.

(45) Yanai, T.; Tew, D. P.; Handy, N. C. A New Hybrid Exchange-correlation Functional Using the Coulomb-Attenuating Method (CAM-B3LYP). Chem. Phys. Lett. 2004, 393 (13), 51-57. https://doi.org/10.1016/j.cplett.2004.06.011.

(46) Tomasi, J.; Mennucci, B.; Cammi, R. Quantum Mechanical Continuum Solvation Models. Chem. Rev. 2005, 105 (8), 2999-3094. https://doi.org/10.1021/cr9904009.

(47) Gaussian 16, Revision A.03, M. J. Frisch, G. W. Trucks, H. B. Schlegel, G. E. Scuseria, M. A. Robb, J. R. Cheeseman, G. Scalmani, V. Barone, G. A. Petersson, H. Nakatsuji, X. Li, M. Caricato, A. V. Marenich, J. Bloino, B. G. Janesko, R. Gomperts, B. Mennucci, H. P. Hratchian, J. V. Ortiz, A. F. Izmaylov, J. L. Sonnenberg, D. Williams-Young, F. Ding, F. Lipparini, F. Egidi, J. Goings, B. Peng, A. Petrone, T. Henderson, D. Ranasinghe, V. G. Zakrzewski, J. Gao, N. Rega, G. Zheng, W. Liang, M. Hada, M. Ehara, K. Toyota, R. Fukuda, J. Hasegawa, M. Ishida, T. Nakajima, Y. Honda, O. Kitao, H. Nakai, T. Vreven, K. Throssell, J. A. Montgomery, Jr., J. E. Peralta, F. Ogliaro, M. J. Bearpark, J. J. Heyd, E. N. Brothers, K. N. Kudin, V. N. Staroverov, T. A. Keith, R. Kobayashi, J. Normand, K. Raghavachari, A. P. Rendell, J. C. Burant, S. S. Iyengar, J. Tomasi, M. Cossi, J. M. Millam, M. Klene, C. Adamo, R. Cammi, J. W. Ochterski, R. L. Martin, K. Morokuma, O. Farkas, J. B. Foresman, and D. J. Fox, Gaussian, Inc., Wallingford CT, 2016.. 